IJJM

Ilomata International Journal of Management

P-ISSN: 2714-8971; E-ISSN:2714-8963

Vol. 1 No. 3 July 2020 pp. 102-112

https://www.ilomata.org/index.php/ijim

\title{
Recruitment Analysis on Employee Performance With Variable Control As Moderating On Manufacturing Company
}

\author{
Nashrudin Setiawan ${ }^{1}$, Emi Wakhyuni ${ }^{2}$, Nur Afrina Siregar ${ }^{3}$ \\ ${ }^{123}$ Management at Pembangunan Pancabudi University of Medan \\ Faculty of Social Science
}

Submitted : July 8, 2020 Revised : July 22, 2020 Published : July 30, 2020

\begin{abstract}
The analysis of this study aims to determine whether recruitment positive and significant effect on the performance of employees in the Manufacturing Company. To know able to moderate recruitment monitoring the performance of employees in the Manufacturing Company. Data analysis technique used is quantitative method with SPSS version 16.0. This study uses regression analysis moderating. The population in this study were all employees Manufacturing Company, and a sample of 44 respondents. The primary data using questionnaires. The results showed that recruitment positive and significant effect on the performance of employees in the Manufacturing Company. Supervision able to moderate recruitment to employee performance on a Manufacturing Company.
\end{abstract}

Keyword: Recruitment, Supervision, Performance.

\section{INTRODUCTION}

Current business conditions, significant improvements. This progress is marked by sophisticated technology that was created. It has an impact on the pace of the world economy, which grew rapidly. However, in practice, this often is foundan issue that causes many companies have failed, either due to the inability to adapt to technological advances, as well as those caused by deficiencies in the work of the human resources that exist in the company. Unable to deny the existence of skilled human resources have a more strategic role in comparison with other resources.

Every form of organization or any company certainly requires humans as a source of labor in carrying out any activities. Humans become planners, perpetrators, and determines the realization of organizational goals. Reciprocation of a company is determined by the active role of all workers in the company. Labor factor which is a variable that can be influenced by the level of ability, willingness, and human settings on all areas and levels of activity.

Qualified human resources in general, was born through a process of training and development of quality. Recognizing that the dominant factor in the sustainability of the company, then in this case the company needs to improve the quality of human resources as well as increasing attention to what is required of employees that will increase employee performance.

Improved performance is needed to create a good employee performance in order to compete and grow the business for employees or human resources is the motor of an undertaking. Every worker has the potential for running the company. This potential should be utilized properly to be able membeikan optimum output. In this regard, special attention is needed in dealing with 
Recruitment Analysis on Employee Performance With Variable Control As Moderating On

Manufacturing Company

Setiawan, Wakhyuni, \& Siregar

employees or human resources so that performance can be increased, With due regard to the recruitment and supervision.

One aspect in enhancing performance employee to achieve the goals set then the recruitment of employees should receive special attention, influence employee recruitment is important for companies to manage, organize, exploit employees so that the company's goal to reached. Recruitment process to find employees in accordance with the capabilities and skills of prospective employees to the company, because with the right recruitment of equivalent or the performance of the employee will fit even in excess of the standard and will make a positive contribution.

Supervision is a process to ensure that the goals of the organization and management reached. surveillance helpful in assessing whether the planning, organizing and directing has been carried out effectively and it is very closely linked to the achievement of objectives that can be seen from the work. Supervision is closely related to the implementation of this work in terms of employee performance so that the work can be accomplished in accordance with a predetermined plan.

Namely recruitment problems policy acceptance test process not in accordance with company guidelines, sometimes employees with the best criteria to be used has not been hired company employees, and forgetting the availability of sufficient time upon receipt of a prospective employee.

\section{METHOD}

Performance is the result of each periodic needs assessment. This is because the employee performance appraisal can then be used as an analysis of the needs of training implementation. Martoyo (2014).

Employee performance that is common to most jobs include the following indicators: the quantity of yield, quality of results, timeliness of results and the ability to cooperate. According Malthis (2013) factors affecting performance are: (1) Work motivation. Conditions or energy that drives self-directed or directed employees to achieve organizational goals of the company; (2) Organizational culture. A pattern of shared basic assumptions learned by groups within an organization as a means to solve the problem; (3) Work environment. Everything that is around the workers who can influence him in carrying out the tasks entrusted; and (4) Working facilities. Means of support in the company's activities in physical form and is used in the normal course of the company.

Hasibuan (2014) states that recruitment is the search for and influence of labor, to want to apply for jobs that exist within an organization. Withdrawal of employees aimed at providing employees enough so that management can choose employees who meet the qualifications they need. According to Hasibuan (2014: 73), an indicator of recruitment is as follows: (1) Identification and Evaluation. The identification phase is the initial stage of the process consists of the determination of the elements was observed; (2) Selection process. Selection is the process of selecting people who have the qualifications needed to fill the job in an organization.; and (3) Placement. Placement is not a final decision. This is an initial step that consists of what according to estimates supervisors concerned can be done by the new workers with what was "requested" by the workers (in the form of intrinsic interest, the opportunity to work together, the possibilities of sale, payment or reward).

Based on expert opinions above, it can be concluded that the indicator for the variable recruitment in this study is policies, procedures and methods. 
Recruitment Analysis on Employee Performance With Variable Control As Moderating On Manufacturing Company

Setiawan, Wakhyuni, \& Siregar

According to Ibrahim (2015: 155), oversight of the work is an activity manager who arranged for the works accomplished in accordance with the established plan and desired results. According to Ibrahim (2015: 160), the regulatory process consists of several actions that are fundamental to all monitoring, namely: (1) Determination Standards Implementation / Planning. The first stage in the implementation of the surveillance is to set the standard, the standard means as a unit of measurement that can be used as a benchmark for assessing the results; (2) Determining Measurement / Assessment Work. Determination of measurement / assessment work is to determine the precise measurement of the implementation of activities; (3) Corrective Action retrieval when needed. When the results of the analysis indicate a corrective action, this action must be taken. Corrective action can be taken in various forms with modified or repaired.

Table 1. Operational Definition of a Variable

\begin{tabular}{|c|c|c|c|}
\hline variables & Operational definition & Indicator & Scale \\
\hline $\begin{array}{l}\text { Recruitment } \\
\text { (X) }\end{array}$ & $\begin{array}{l}\text { The search process and } \\
\text { "enchantment" job } \\
\text { candidates (applicants) who } \\
\text { are able to apply as an } \\
\text { employee. }\end{array}$ & $\begin{array}{l}\text { 1. Your organization's policy } \\
\text { 2. plan } \\
\text { 3. method } \\
\text { 4. procedure } \\
\text { 5. execution time } \\
\text { 6. recruitment sources } \\
\text { 7. obstacles }\end{array}$ & Likert scale \\
\hline $\begin{array}{l}\text { Supervision } \\
(\mathrm{Z})\end{array}$ & $\begin{array}{l}\text { Event managers who try to } \\
\text { get the jobs done in } \\
\text { accordance with the } \\
\text { established plan and desired } \\
\text { results. }\end{array}$ & $\begin{array}{l}\text { 1. accurate } \\
\text { 2. on time } \\
\text { 3. objective } \\
\text { 4. realistic } \\
\text { 5. coordinated }\end{array}$ & Likert scale \\
\hline $\begin{array}{l}\text { Performance } \\
\text { Employee } \\
\text { (Y) }\end{array}$ & $\begin{array}{l}\text { The results of the quality and } \\
\text { quantity of work achieved by } \\
\text { an employee in performing } \\
\text { their duties in accordance } \\
\text { with the responsibilities } \\
\text { given to him. }\end{array}$ & $\begin{array}{l}\text { 1. Quality } \\
\text { 2. Quantity } \\
\text { 3. Timeliness of results. } \\
\text { 4. Effectiveness } \\
\text { 5. autonomy } \\
\text { 6. work commitment }\end{array}$ & Likert scale \\
\hline
\end{tabular}

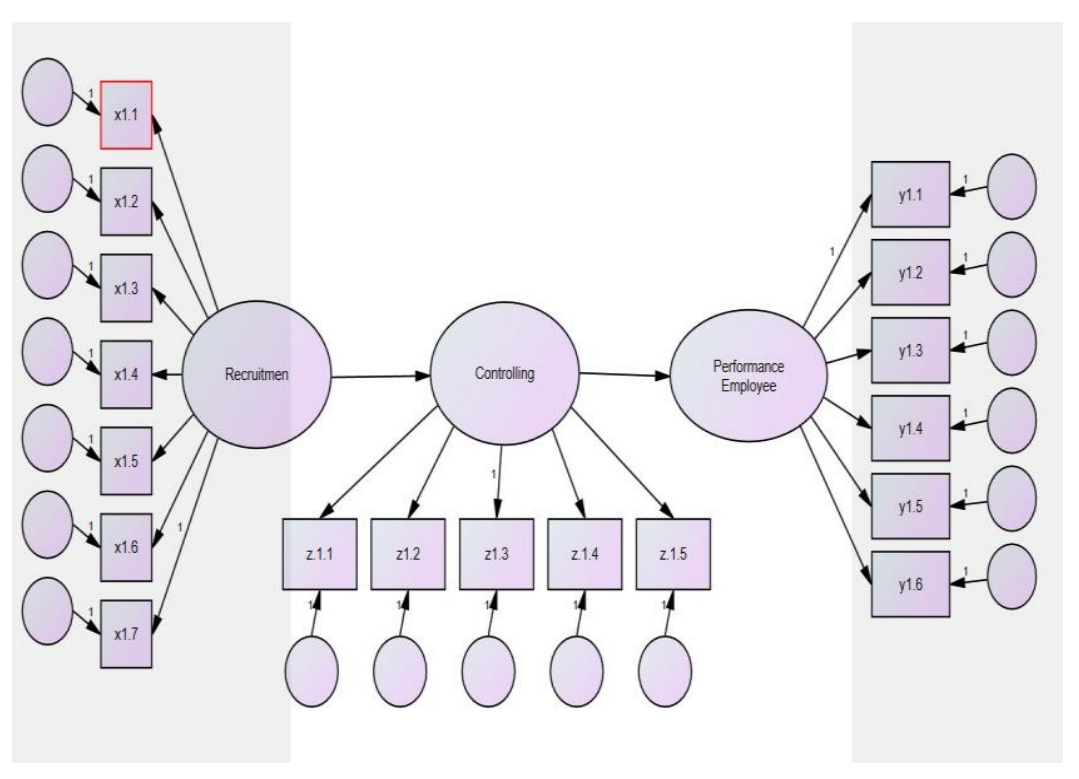

Figure 1. Research frame work 
Recruitment Analysis on Employee Performance With Variable Control As Moderating On

Manufacturing Company

Setiawan, Wakhyuni, \& Siregar

\section{RESULT AND DISCUSSION}

Table 2. Assessment of Respondents Against Policy Indicators (X.1)

\begin{tabular}{|c|c|c|c|c|c|c|}
\hline \multirow{3}{*}{$\begin{array}{l}\text { Respondents } \\
\text { answer }\end{array}$} & \multicolumn{6}{|c|}{ item Questions } \\
\hline & \multicolumn{2}{|c|}{$\begin{array}{l}\text { The Company has been } \\
\text { engaged in the recruitment } \\
\text { process according to } \\
\text { company policy }\end{array}$} & \multicolumn{2}{|c|}{$\begin{array}{c}\text { Companies recruit } \\
\text { employees in accordance } \\
\text { with the criteria required } \\
\text { positions companies }\end{array}$} & \multicolumn{2}{|c|}{$\begin{array}{l}\text { The company carries out } \\
\text { the process of recruitment } \\
\text { tests in accordance with } \\
\text { existing policy }\end{array}$} \\
\hline & Frequency & $\%$ & Frequency & $\%$ & Frequency & $\%$ \\
\hline $\begin{array}{l}\text { Strongly } \\
\text { Disagree }\end{array}$ & - & - & - & - & - & - \\
\hline Disagree & 3 & 6.8 & 11 & 25.0 & 8 & 18.2 \\
\hline Doubtful & 18 & 40.9 & 10 & 22.7 & 14 & 31.8 \\
\hline Agree & 18 & 40.9 & 23 & 52.3 & 19 & 43.2 \\
\hline Strongly agree & 5 & 11.4 & - & - & 3 & 6.8 \\
\hline Total & 44 & 100 & 44 & 100 & 44 & 100 \\
\hline mean & \multicolumn{2}{|c|}{3.5682} & \multicolumn{2}{|c|}{3.2727} & \multicolumn{2}{|c|}{3.3864} \\
\hline
\end{tabular}

Table 3. Assessment Procedure Respondents Against Indicators (X.2)

\begin{tabular}{|c|c|c|c|c|c|c|}
\hline \multirow{3}{*}{$\begin{array}{l}\text { Respondents } \\
\text { answer }\end{array}$} & \multicolumn{6}{|c|}{ item Questions } \\
\hline & \multicolumn{2}{|c|}{$\begin{array}{l}\text { Companies no charge } \\
\text { during the recruitment } \\
\text { process runs }\end{array}$} & \multicolumn{2}{|c|}{$\begin{array}{l}\text { The Company does not } \\
\text { charge to employees } \\
\text { during the recruitment } \\
\text { process underway }\end{array}$} & \multicolumn{2}{|c|}{$\begin{array}{l}\text { Companies recruit } \\
\text { employees in accordance } \\
\text { with the best criteria }\end{array}$} \\
\hline & Frequency & $\%$ & Frequency & $\%$ & Frequency & $\%$ \\
\hline $\begin{array}{l}\text { Strongly } \\
\text { Disagree }\end{array}$ & - & - & - & - & - & - \\
\hline Disagree & 13 & 29.5 & 24 & 54.5 & 12 & 27.3 \\
\hline Doubtful & 13 & 29.5 & 4 & 9.1 & 16 & 36.4 \\
\hline Agree & 14 & 31.8 & 6 & 13.6 & 14 & 31.8 \\
\hline Strongly agree & 4 & 9.1 & 10 & 22.7 & 2 & 4.5 \\
\hline Total & 44 & 100 & 44 & 100 & 44 & 100 \\
\hline mean & \multicolumn{2}{|c|}{3.2045} & \multicolumn{2}{|c|}{3.0455} & \multicolumn{2}{|c|}{3.1364} \\
\hline
\end{tabular}

Table 4. Assessment Indicators Respondents Against Method (X.3)

\begin{tabular}{|c|c|c|c|c|c|c|}
\hline \multirow{3}{*}{$\begin{array}{l}\text { Respondents } \\
\text { answer }\end{array}$} & \multicolumn{6}{|c|}{ item Questions } \\
\hline & \multicolumn{2}{|c|}{$\begin{array}{l}\text { Companies always provide } \\
\text { sufficient time admission of } \\
\text { candidates }\end{array}$} & \multicolumn{2}{|c|}{$\begin{array}{l}\text { The company delivers the } \\
\text { right time for each stage of } \\
\text { the selection recruitment }\end{array}$} & \multicolumn{2}{|c|}{$\begin{array}{l}\text { The Company provides } \\
\text { recruiting information } \\
\text { openly }\end{array}$} \\
\hline & Frequency & $\%$ & Frequency & $\%$ & Frequency & $\%$ \\
\hline $\begin{array}{l}\text { Strongly } \\
\text { Disagree }\end{array}$ & - & - & - & - & - & - \\
\hline Disagree & 15 & 34.1 & 8 & 18.2 & 9 & 20.5 \\
\hline Doubtful & 19 & 43.2 & 14 & 31.8 & 22 & 50.0 \\
\hline Agree & 7 & 15.9 & 19 & 43.2 & 11 & 25.0 \\
\hline Strongly agree & 3 & 6.8 & 3 & 6.8 & 2 & 4.5 \\
\hline Total & 44 & 100 & 44 & 100 & 44 & 100 \\
\hline mean & \multicolumn{2}{|c|}{2.9545} & \multicolumn{2}{|c|}{3.3864} & \multicolumn{2}{|c|}{3.1364} \\
\hline
\end{tabular}


Recruitment Analysis on Employee Performance With Variable Control As Moderating On

Manufacturing Company

Setiawan, Wakhyuni, \& Siregar

Table 5. Respondents Rate Against Indicators Determination Standards Implementation / Planning (Z.1)

\begin{tabular}{|c|c|c|c|c|c|c|}
\hline \multirow{3}{*}{$\begin{array}{l}\text { Respondents } \\
\text { answer }\end{array}$} & \multicolumn{6}{|c|}{ item Questions } \\
\hline & \multicolumn{2}{|c|}{$\begin{array}{c}\text { The company set the } \\
\text { standard of work of each to } \\
\text { employees }\end{array}$} & \multicolumn{2}{|c|}{$\begin{array}{l}\text { Employees follow the rules } \\
\text { clock in and clock home } \\
\text { from work in accordance } \\
\text { with company standards }\end{array}$} & \multicolumn{2}{|c|}{$\begin{array}{c}\text { Employees carry out tasks } \\
\text { that have been determined } \\
\text { leaders based on job } \\
\text { description }\end{array}$} \\
\hline & Frequency & $\%$ & Frequency & $\%$ & Frequency & $\%$ \\
\hline $\begin{array}{l}\text { Strongly } \\
\text { Disagree }\end{array}$ & - & - & 5 & 11.4 & 1 & 2.3 \\
\hline Disagree & 3 & 6.8 & 2 & 4.5 & 5 & 11.4 \\
\hline Doubtful & 9 & 20.5 & 9 & 20.5 & 4 & 9.1 \\
\hline Agree & 12 & 27.3 & 11 & 25.0 & 19 & 43.2 \\
\hline Strongly agree & 20 & 45.5 & 17 & 38.6 & 15 & 34.1 \\
\hline Total & 44 & 100 & 44 & 100 & 44 & 100 \\
\hline mean & \multicolumn{2}{|c|}{4.1136} & \multicolumn{2}{|c|}{3.7500} & \multicolumn{2}{|c|}{3.9545} \\
\hline
\end{tabular}

Table 6. Respondents Rate Against Indicators determination of Measurement / Assessment Work (Z.2)

\begin{tabular}{|c|c|c|c|c|c|c|}
\hline \multirow{3}{*}{$\begin{array}{l}\text { Respondents } \\
\text { answer }\end{array}$} & \multicolumn{6}{|c|}{ item Questions } \\
\hline & \multicolumn{2}{|c|}{$\begin{array}{l}\text { Checking the results - the } \\
\text { work done by all employees }\end{array}$} & \multicolumn{2}{|c|}{$\begin{array}{c}\text { Measure / compare the } \\
\text { work with the standards } \\
\text { set }\end{array}$} & \multicolumn{2}{|c|}{$\begin{array}{l}\text { Leaders give feedback to } \\
\text { the father / mother on } \\
\text { the assessment of work }\end{array}$} \\
\hline & Frequency & $\%$ & Frequency & $\%$ & Frequency & $\%$ \\
\hline $\begin{array}{l}\text { Strongly } \\
\text { Disagree }\end{array}$ & - & - & 2 & 4.5 & 7 & 15.9 \\
\hline Disagree & 16 & 36.4 & 3 & 6.8 & 16 & 36.4 \\
\hline Doubtful & 1 & 2.3 & 7 & 15.9 & 3 & 6.8 \\
\hline Agree & 6 & 13.6 & 8 & 18.2 & 7 & 15.9 \\
\hline Strongly agree & 21 & 47.7 & 24 & 54.5 & 11 & 25.0 \\
\hline Total & 44 & 100 & 44 & 100 & 44 & 100 \\
\hline mean & \multicolumn{2}{|c|}{3.7273} & \multicolumn{2}{|c|}{4.1136} & \multicolumn{2}{|c|}{2.9773} \\
\hline
\end{tabular}

Table 7. Respondents Rate Against Indicators Capturing action Correction When Necessary (Z.3)

\begin{tabular}{|l|c|c|c|c|c|c|}
\hline \multirow{2}{*}{$\begin{array}{c}\text { Respondents } \\
\text { answer }\end{array}$} & $\begin{array}{r}\text { Supervision is done to } \\
\text { avoid misuse / diversion / } \\
\text { mistakes made by } \\
\text { employees }\end{array}$ & $\begin{array}{c}\text { When you make a } \\
\text { mistake in carrying out } \\
\text { the discharge of duties, } \\
\text { leadership will be } \\
\text { reprimanded for repair }\end{array}$ & $\begin{array}{c}\text { Leaders always provide a } \\
\text { solution / remedial } \\
\text { action to the father / } \\
\text { mother if something } \\
\text { goes wrong }\end{array}$ \\
\cline { 2 - 8 } & Frequency & $\%$ & Frequency & $\%$ & Frequency & $\%$ \\
\hline $\begin{array}{l}\text { Strongly } \\
\text { Disagree }\end{array}$ & - & - & - & - & - & - \\
\hline Disagree & 7 & 15.9 & 8 & 18.2 & 8 & 18.2 \\
\hline Doubtful & 5 & 11.4 & 12 & 27.3 & 7 & 15.9 \\
\hline Agree & 14 & 31.8 & 8 & 18.2 & 4 & 9.1 \\
\hline Strongly agree & 44 & 40.9 & 16 & 36.4 & 25 & 56.8 \\
\hline Total & 18 & 44 & 100 & 44 & 100 \\
\hline mean & 3.9773 & \multicolumn{7}{|c|}{3.7273} & & 4.0455 \\
\hline
\end{tabular}


Recruitment Analysis on Employee Performance With Variable Control As Moderating On

Manufacturing Company

Setiawan, Wakhyuni, \& Siregar

Table 8. Respondents Rate Against Indicators Quality (Y.1)

\begin{tabular}{|c|c|c|c|c|c|c|}
\hline \multirow{3}{*}{$\begin{array}{l}\text { Respondents } \\
\text { answer }\end{array}$} & \multicolumn{6}{|c|}{ item Questions } \\
\hline & \multicolumn{2}{|c|}{$\begin{array}{l}\text { Manufacturing companies } \\
\text { pay attention to the quality } \\
\text { standards of employees that } \\
\text { exceed the quality standards } \\
\text { of other existing employees }\end{array}$} & \multicolumn{2}{|c|}{$\begin{array}{l}\text { Manufacturing companies } \\
\text { pay attention to } \\
\text { employees so that } \\
\text { employees can get the job } \\
\text { done with no rush }\end{array}$} & \multicolumn{2}{|c|}{$\begin{array}{l}\text { Manufacturing companies } \\
\text { pay attention to employees in } \\
\text { order to complete the task in } \\
\text { accordance responsibilities }\end{array}$} \\
\hline & Frequency & $\%$ & Frequency & $\%$ & Frequency & $\%$ \\
\hline Strongly Disagree & - & - & - & - & - & - \\
\hline Disagree & 6 & 13.6 & 8 & 18.2 & 4 & 9.1 \\
\hline Doubtful & 23 & 52.3 & 14 & 31.8 & 21 & 47.7 \\
\hline Agree & 13 & 29.5 & 19 & 43.2 & 18 & 40.9 \\
\hline Strongly agree & 2 & 4.5 & 3 & 6.8 & 1 & 2.3 \\
\hline Total & 44 & 100 & 44 & 100 & 44 & 100 \\
\hline Mean & \multicolumn{2}{|c|}{3.2500} & \multicolumn{2}{|c|}{3.3864} & \multicolumn{2}{|c|}{3.3636} \\
\hline
\end{tabular}

Table 9. Respondents Rate Against Indicators Quantity (Y.2)

\begin{tabular}{|c|c|c|c|c|c|c|}
\hline \multirow{3}{*}{$\begin{array}{l}\text { Respondents } \\
\text { answer }\end{array}$} & \multicolumn{6}{|c|}{ item Questions } \\
\hline & \multicolumn{2}{|c|}{$\begin{array}{l}\text { Manufacturing companies } \\
\text { pay attention to the } \\
\text { employees to always work in } \\
\text { accordance with the number } \\
\text { of targeted organizations }\end{array}$} & \multicolumn{2}{|c|}{$\begin{array}{l}\text { Manufacturing company always } \\
\text { pay attention to the employees } \\
\text { so that employees are able to } \\
\text { work in accordance with the } \\
\text { standards organization }\end{array}$} & \multicolumn{2}{|c|}{$\begin{array}{l}\text { Manufacturing company always } \\
\text { pay attention to the employees } \\
\text { so that employees always carry } \\
\text { out duties in accordance with } \\
\text { the command leadership }\end{array}$} \\
\hline & Frequency & $\%$ & Frequency & $\%$ & Frequency & $\%$ \\
\hline Strongly Disagree & - & - & - & - & - & - \\
\hline Disagree & 15 & 34.1 & 8 & 18.2 & 13 & 29.5 \\
\hline Doubtful & 13 & 29.5 & 13 & 29.5 & 20 & 45.5 \\
\hline Agree & 14 & 31.8 & 17 & 38.6 & 11 & 25.0 \\
\hline Strongly agree & 2 & 4.5 & 6 & 13.6 & - & - \\
\hline Total & 44 & 100 & 44 & 100 & 44 & 100 \\
\hline mean & \multicolumn{2}{|c|}{3.0682} & \multicolumn{2}{|c|}{3.4773} & \multicolumn{2}{|c|}{2.9545} \\
\hline
\end{tabular}

Table 10. Against Respondents Rate Indicators Timeliness Of Results (Y.3)

\begin{tabular}{|c|c|c|c|c|c|c|}
\hline \multirow{3}{*}{$\begin{array}{l}\text { Respondents } \\
\text { answer }\end{array}$} & \multicolumn{6}{|c|}{ item Questions } \\
\hline & \multicolumn{2}{|c|}{$\begin{array}{c}\text { Manufacturing } \\
\text { companyalways pay } \\
\text { attention to the employees } \\
\text { so that employees can work } \\
\text { according to standards set } \\
\text { time }\end{array}$} & \multicolumn{2}{|c|}{$\begin{array}{c}\text { Manufacturing } \\
\text { companyalways pay } \\
\text { attention to the employee } \\
\text { for the employee to } \\
\text { complete the job with a } \\
\text { fixed time }\end{array}$} & \multicolumn{2}{|c|}{$\begin{array}{c}\text { Manufacturing } \\
\text { companyalways pay } \\
\text { attention to the employees } \\
\text { so that employees feel } \\
\text { satisfied when my work } \\
\text { done with the right timing }\end{array}$} \\
\hline & Frequency & $\%$ & Frequency & $\%$ & Frequency & $\%$ \\
\hline Strongly Disagree & - & - & - & - & - & - \\
\hline Disagree & 13 & 29.5 & 8 & 18.2 & 6 & 13.6 \\
\hline Doubtful & 22 & 50.0 & 14 & 31.8 & 20 & 45.5 \\
\hline Agree & 9 & 20.5 & 20 & 45.5 & 14 & 31.8 \\
\hline Strongly agree & - & - & 2 & 4.5 & 4 & 9.1 \\
\hline Total & 44 & 100 & 44 & 100 & 44 & 100 \\
\hline mean & \multicolumn{2}{|c|}{2.9091} & \multicolumn{2}{|c|}{3.3636} & \multicolumn{2}{|c|}{3.3636} \\
\hline
\end{tabular}


Recruitment Analysis on Employee Performance With Variable Control As Moderating On Manufacturing Company

Setiawan, Wakhyuni, \& Siregar

Table 11. Uji Validity (X) Recruitment

Item-Total Statistics

\begin{tabular}{|l|r|r|r|r|}
\hline & $\begin{array}{c}\text { Scale Mean if Item } \\
\text { Deleted }\end{array}$ & $\begin{array}{c}\text { Scale Variance if } \\
\text { Item Deleted }\end{array}$ & $\begin{array}{c}\text { Corrected Item- } \\
\text { Total Correlation }\end{array}$ & $\begin{array}{c}\text { Cronbach's Alpha } \\
\text { if Item Deleted }\end{array}$ \\
\hline statement X.1 & 25.5227 & 24674 & $\mathbf{. 3 4 4}$ & .819 \\
statement X.2 & 25.8182 & 24106 & $\mathbf{. 3 8 2}$ & .815 \\
statement X.3 & 25.7045 & 22073 & $\mathbf{. 6 3 3}$ & .787 \\
statement X.4 & 25.8864 & 22010 & $\mathbf{. 5 4 7}$ & .797 \\
statement X.5 & 26.0455 & 19626 & $\mathbf{. 5 9 7}$ & .794 \\
statement X.6 & 25.9545 & 23765 & $\mathbf{. 4 0 3}$ & .813 \\
statement X.7 & 26.1364 & 21655 & $\mathbf{. 6 7 1}$ & .782 \\
statement x.8 & 25.7045 & 22073 & $\mathbf{. 6 3 3}$ & .787 \\
statement X.9 & 25.9545 & 23579 & $\mathbf{. 4 9 0}$ & .804 \\
\hline
\end{tabular}

Table 12. Test Validity (Z) Supervision

Item-Total Statistics

\begin{tabular}{|l|r|r|r|r|}
\hline & $\begin{array}{c}\text { Scale Mean if Item } \\
\text { Deleted }\end{array}$ & $\begin{array}{c}\text { Scale Variance if } \\
\text { Item Deleted }\end{array}$ & $\begin{array}{c}\text { Corrected Item- } \\
\text { Total Correlation }\end{array}$ & $\begin{array}{c}\text { Cronbach's Alpha } \\
\text { if Item Deleted }\end{array}$ \\
\hline statement Z.1 & 30.2727 & 48296 & $\mathbf{. 6 8 2}$ & .849 \\
statement Z.2 & 30.6364 & 49121 & $\mathbf{. 4 0 4}$ & .873 \\
statement Z.3 & 30.4318 & 47832 & $\mathbf{. 6 4 9}$ & .850 \\
statement Z.4 & 30.6591 & 43951 & $\mathbf{. 6 8 3}$ & .846 \\
statement Z.5 & 30.2727 & 47273 & $\mathbf{. 5 9 8}$ & .854 \\
statement Z.6 & 31.4091 & 44712 & $\mathbf{. 5 7 9}$ & .858 \\
statement Z.7 & 30.4091 & 47085 & $\mathbf{. 6 8 0}$ & .847 \\
statement Z.8 & 30.6591 & 46974 & $\mathbf{. 6 4 4}$ & .850 \\
statement Z.9 & 30.3409 & 47114 & $\mathbf{. 5 8 7}$ & .855 \\
\hline
\end{tabular}

Table 13. Test Validity (Y) Employee Performance

Item-Total Statistics

\begin{tabular}{|l|r|r|r|r|}
\hline & $\begin{array}{c}\text { Scale Mean if Item } \\
\text { Deleted }\end{array}$ & $\begin{array}{c}\text { Scale Variance if } \\
\text { Item Deleted }\end{array}$ & $\begin{array}{c}\text { Corrected Item- } \\
\text { Total Correlation }\end{array}$ & $\begin{array}{c}\text { Cronbach's Alpha } \\
\text { if Item Deleted }\end{array}$ \\
\hline statement Y.1 & 25.8864 & 27219 & $\mathbf{. 5 8 9}$ & .914 \\
statement Y.2 & 25.7500 & 24564 & $\mathbf{. 8 2 3}$ & .898 \\
statement Y.3 & 25.7727 & 26970 & $\mathbf{. 6 9 7}$ & .908 \\
statement Y.4 & 26.0682 & 24856 & $\mathbf{. 7 2 5}$ & .906 \\
statement Y.5 & 25.6591 & 24276 & $\mathbf{. 7 6 9}$ & .902 \\
statement Y.6 & 26.1818 & 27222 & $\mathbf{. 5 9 4}$ & .914 \\
statement Y.7 & 26.2273 & 26831 & $\mathbf{. 6 8 9}$ & .908 \\
statement Y.8 & 25.7727 & 24738 & $\mathbf{. 8 3 5}$ & .898 \\
statement Y.9 & 25.7727 & 26133 & $\mathbf{. 6 5 0}$ & .911 \\
\hline
\end{tabular}

Table 14. Uji Reliability (X) Recruitment

Reliability Statistics

\begin{tabular}{|r|r|}
\hline Cronbach's Alpha & N of Items \\
\hline .819 & 9 \\
\hline
\end{tabular}


Recruitment Analysis on Employee Performance With Variable Control As Moderating On Manufacturing Company

Setiawan, Wakhyuni, \& Siregar

Table 15 Test Reliability (Z) Supervision

Reliability Statistics

\begin{tabular}{|r|r|}
\hline Cronbach's Alpha & N of Items \\
\hline .868 & 9 \\
\hline
\end{tabular}

Table 16. Uji Reliability (Y)Employee performance

Reliability Statistics

\begin{tabular}{|r|r|}
\hline Cronbach's Alpha & N of Items \\
\hline .916 & 9 \\
\hline
\end{tabular}

Histogram

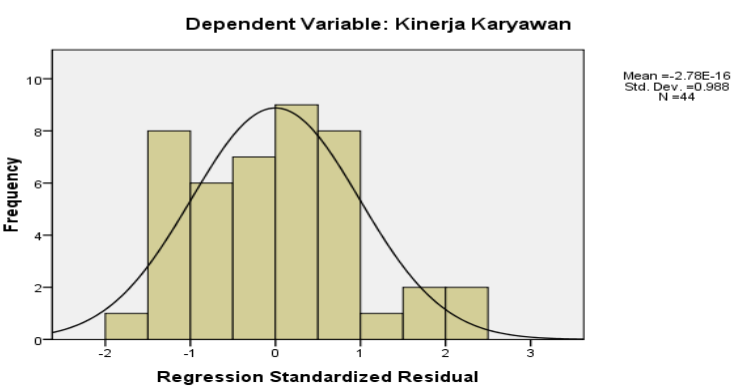

Figure 2. Histograms Normality Test

Normal P-P Plot of Regression Standardized Residual

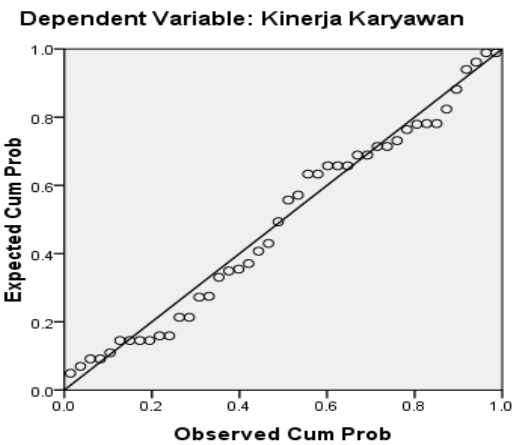

Figure 3. PP plot Normality Test

Table 17. Normality Test One Sample Kolmogorov-Smirnov Test

One-Sample Kolmogorov-Smirnov Test

\begin{tabular}{|c|c|c|}
\hline & & $\begin{array}{c}\text { Residual } \\
\text { unstandardized }\end{array}$ \\
\hline \multicolumn{2}{|l|}{$\mathrm{N}$} & 44 \\
\hline \multirow[t]{2}{*}{ normal Parametersa } & mean & .0000000 \\
\hline & Std. deviation & 3.77367326 \\
\hline \multirow[t]{3}{*}{ Most Extreme Differences } & Absolute & .094 \\
\hline & positive & .094 \\
\hline & negative & -.089 \\
\hline Kolmogorov-Smirnov Z & & .623 \\
\hline Asymp. Sig. (2-tailed) & & .832 \\
\hline
\end{tabular}


Recruitment Analysis on Employee Performance With Variable Control As Moderating On Manufacturing Company

Setiawan, Wakhyuni, \& Siregar

Scatterplot

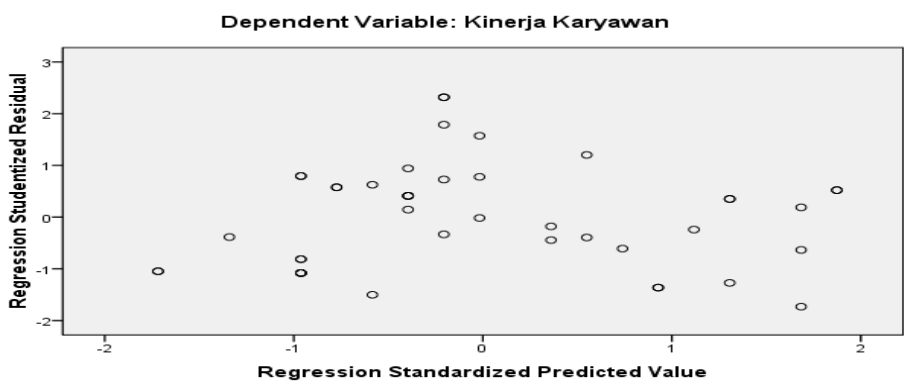

Figure 4. Scatterplot Test Heteroskidastity

Table 18. Simple Linear Regression

Coefficientsa

\begin{tabular}{|c|c|c|c|c|c|c|c|}
\hline \multirow{2}{*}{\multicolumn{2}{|c|}{ Model }} & \multicolumn{2}{|c|}{$\begin{array}{l}\text { Coefficients } \\
\text { unstandardized }\end{array}$} & \multirow[b]{2}{*}{$\mathrm{t}$} & \multirow[b]{2}{*}{ Sig. } & \multicolumn{2}{|c|}{ collinearity Statistics } \\
\hline & & $\mathrm{B}$ & Std. Error & & & tolerance & VIF \\
\hline 1 & (Constant) & 5705 & 3253 & 1,754 & .087 & & \\
\hline & recruitment & .805 & .110 & 7318 & .000 & 1,000 & 1,000 \\
\hline
\end{tabular}

a. Dependent Variable: Employee Performance

Table 19. Partial Test

Coefficientsa

\begin{tabular}{|c|c|c|c|c|c|c|c|}
\hline \multirow{2}{*}{\multicolumn{2}{|c|}{ Model }} & \multicolumn{2}{|c|}{$\begin{array}{c}\text { Coefficients } \\
\text { unstandardized }\end{array}$} & \multirow[b]{2}{*}{$\mathrm{t}$} & \multirow[b]{2}{*}{ Sig. } & \multicolumn{2}{|c|}{ collinearity Statistics } \\
\hline & & B & Std. Error & & & tolerance & VIF \\
\hline \multirow[t]{2}{*}{1} & (Constant) & 5705 & 3253 & 1,754 & .087 & & \\
\hline & recruitment & .805 & .110 & 7318 & .000 & 1,000 & 1,000 \\
\hline
\end{tabular}

a. Dependent Variable: Employee Performance

Table 20. Coefficient Determination

Model Summaryb

\begin{tabular}{|l|r|r|r|r|}
\hline Model & \multicolumn{1}{|c|}{$\mathrm{R}$} & \multicolumn{1}{|c|}{ R Square } & \multicolumn{1}{|c|}{ Adjusted R Square } & \multicolumn{1}{|c|}{ Std. Error of the Estimate } \\
\hline 1 & $.749 \mathrm{a}$ & .560 & $\mathbf{. 5 5 0}$ & 3.81833 \\
\hline
\end{tabular}

a. Predictors: (Constant), Recruitment

b. Dependent Variable: Employee Performance

Table 21. Test Moderating Regression Analysis (MRA)

Coefficientsa

\begin{tabular}{|cl|r|r|r|r|}
\hline \multirow{2}{*}{ Model } & & \multicolumn{2}{|c|}{ Coefficients unstandardized } & \multicolumn{2}{|c|}{} \\
\cline { 3 - 4 } & \multicolumn{2}{|c|}{$\mathrm{B}$} & Std. Error & $\mathrm{t}$ & \multicolumn{2}{|c|}{ Sig. } \\
\hline 1 & (Constant) & 2,959 & 1,658 & 1,785 & .082 \\
& Employee performance & .006 & .056 & 4,102 & .019 \\
\hline
\end{tabular}

a. Dependent Variable: AbsRes_1 
Recruitment Analysis on Employee Performance With Variable Control As Moderating On

Manufacturing Company

Setiawan, Wakhyuni, \& Siregar

CONCLUSION

\section{Influence Recruitment on Employee Performance}

The results showed that recruitment in Manufacturing Company, affect the performance of employees. This can be seen from multiple linear regression analysis through the $t$ test is positive with value amounted to 7.318 with sig. 0,000 . Based on these results, it can be concluded that the hypothesis is 1 (one) in this study tested and acceptable. Positive direction shows that whenever there is increased recruitment conducted by the Manufacturing Company, will lead to increased performance simultaneously Manufacturing Company employees by 0, 805unit. In other words, when recruitment Manufacturing Company which consists of policies, procedures and methods, the performance of employees of PT. Eaglewood Putra Mandiri Steel will increase as well. The results are consistent with research Yani (2017), Aziz (2017) and Jaya (2018), indicate that recruitment positive and significant effect on employee performance.

The results are consistent with the objectives of the research to know whether the recruitment positive and significant effect on the performance of employees at the Company Manufacturing located in Medan has been accomplished and also has completed / answer the problems that exist in the identification of problem points No. 1, 2 and 3, namely employees received not use the system of recruitment / guidelines for the desired company. Employees who are in the recruitment is not specific to the operation should run the company. Employees who in recruitment is not in accordance with the desired company is already missed.

\section{Recruitment Influence on Performance Monitoring For Variable With Moderating}

The results showed that the test moderating with absolutely residual value, it is known that the value is 0.006 and significant regression $0.019<0.05$. These results can be concluded that significant oversight as moderating variable.

$$
\begin{aligned}
& Z=2,959+0,006 X+\varepsilon_{1} \\
& Y=5.705+0.805 X+0.006 Z+\varepsilon_{2}
\end{aligned}
$$

Interpretation of moderating equation is:

a. If everything on the independent variables considered zero then the value of supervision $(\mathrm{Z})$ is equal to 2,959 .

b. If an increase in the recruitment of 1 , then the control $(Z)$ will increase by 0,006 .

c. If everything on the independent variables considered zero then the value of employee performance $(\mathrm{Y})$ is equal to 5.705 .

d. If an increase in the recruitment of 1 , the performance $(\mathrm{Y})$ will increase by 0.805 .

e. If an increase in oversight 1 , then the performance $(\mathrm{Y})$ will increase by 0,006 .

The results are consistent with research Yani (2017), shows that the surveillance as moderating variable. The results are consistent with the purpose of the study to determine whether scrutiny as moderating variable on Manufacturing Company has been accomplished and also has completed / answer the problems that exist in the identification of problems point no 4, 5 and 6 that the employee has not set standards of work execution. Employees have yet to determine the conduct of the assessment work activities appropriately. Employees have not been able to take corrective action in the implementation of the work has been answered. 
Recruitment Analysis on Employee Performance With Variable Control As Moderating On

Manufacturing Company

Setiawan, Wakhyuni, \& Siregar

\section{REFERENCE}

Adnan celik, R. I. (2014). The effects of employee empowerment applications on organizational creativity and innovativeness inenterpriseses: the case of Oiz. European scientific journal, 10, 99-107.

Chen, k. P. (2011). A study on the impact of empowerment on employee performance in the automotive industry in Malaysia.

Gaudreau Meyerson, b. D. (2012). Effect of empowerment on employees performance. Advanced research in economic and management sciences (arems), 2, 40-46.

Pd.suresh, d. J. (2015). Impact of employee empowerment on organizational performance in the case of the automobile industry chennai city. Ijiset - international journal of innovative science, engineering and technology, 2 (4), 20-31.

Shadi ebrahimi mehrabani, m. S. (2013). Relationship between employee empowerment and employee effectiveness. Service science and management research (ssmr), 2 (4), 60-68.

Sofyan, M. (2019). Faktor-Faktor yang Mempengaruhi Jiwa Kewirausahaan. JMK Jurnal Manajemen dan Kewirausabaan), 4(3), 194-204. doi:10.32503/jmk.v4i3.586.

Silvia c. Peters, e. M. (2008). The impact of employee empowerment on service. Mälardalen University, 8-71. 